\title{
Correction of radiation pattern due to antenna reflector deformation in passive monopulse direction finding problem
}

\author{
Vladimir Fidelman ${ }^{1}$, Oleg Morozov ${ }^{1}$, and Yuriy Chumankin ${ }^{1, *}$ \\ ${ }^{1}$ Lobachevsky State University of Nizhny Novgorod, 603022 Gagarin ave. 23, Nizhny Novgorod, \\ Russia
}

\begin{abstract}
Parabolic antenna reflector deformation caused by one-sided heating may be described by linear stretching model. The modeling of the influence of the antenna reflector stretching on the error in determining the bearing in the problem of passive monopulse direction finding was done. The method of electric axis displacement accounting was proposed and its feasibility was shown. The comparison of the probabilities of the bearing estimation with an error less than fixed threshold for the initial and refined radiation pattern was made.
\end{abstract}

\section{Introduction}

The task of passive radio source localization by single receiver is important for space communication and radar systems. Algorithms for the task [1,2] involve the use of a single multi-beam large-sized antenna with several receiving channels. Each receiving channel corresponds to one beam of the antenna radiation pattern (RP). The electrical axes of the various beams are differently oriented. The main step solving the task is direction finding. It is usually assumed that a radio source is active at the direction-finding time. According to the received signal realizations in various channels of the antenna system, the signal amplitudes $\mathrm{A}_{i}$ are estimated, which are then used together with the information about the radiation patterns for calculating the bearing.

The traditional radio source bearing determining algorithms using the single-pulse method [1] work on the linear section of the direction-finding characteristic which is certain function of the RP main beams, using approximation of the RP by smooth functions (for example, Gaussian). In practice, RPs are multi-petal which significantly complicates the bearing estimation task. Receiving a signal from directions corresponding to the side RP petals significantly increases the bearing error relatively to receiving a signal from the main petal [2]. The task is complicated by the fact that during detection and direction finding by the single-pulse method, the error in the RP values contributes to the bearing error.

There are many factors, that lead to distortion of reflector profile during antenna system exploitation, and as result, distortion of radiation pattern. Currently, there are tools to estimate the certain points of the reflector displacement from the initial position [3]. The

*Corresponding author: chumankinyuriy@nifti.unn.ru 
influence of the reflector deformation may be considered when calculating the radiation pattern using classical methods [4]. However, such a task requires significant time and computational resources. More computationally efficient algorithms are proposed for the deformation of the antenna reflector which does not lead to a fundamental change in the profile of the beam. It is advisable to use a quick calculation method for the original diagram corrections. For example, in [5] a method for compensating the reflector deformation was proposed, based on the approximation of the value of the antenna electric axis displacement depending on the reflector rotation.

Various methods for changing the radiation pattern or assessing the antenna deformation were proposed in the literature. Still, computationally efficient algorithms for estimating change in the radiation pattern from the antenna deformation are important for decreasing the bearing estimation error. In the article such algorithm based on reflector stretching model is proposed and its feasibility is shown. The research of the accuracy of direction finding using the initial and refined radiation pattern was done.

\section{Used methods and approximations}

To achieve the goals, it is necessary to solve accessory tasks: calculation of the radiation pattern, signal registration simulation, bearing estimation. Solving the accessory tasks allows preparing data for RP correction method and evaluating the quality of the method.

The implementation of accurate methods for solving the RP calculation task is associated with large computational costs. In some cases, it is advisable to use various simplifications to analyze the scattering field at objects of complex spatial configuration. In this article the reflective point method [6] was used. A discrete representation of the continuous surface of the antenna reflector is considered. Under such conditions radiation pattern is a superposition of the fields created by elements of the reflector, considering their amplitudes and phases.

For simulation, an antenna with a reflector aperture diameter of $D=10 \mathrm{~m}$ and focal length of $F=7 \mathrm{~m}$ was considered. Also, typical 16-irradiator configuration was used for simulation. Irradiator frequency $f$ was taken as $8 \mathrm{GHz}$. RP and irradiator position described in coordinate system consisting from azimuth $\varphi$ and elevation $\theta$. Radiation pattern discretized on the grid of nodes in specified coordinate system. Radiation pattern calculated by the reflective point method is shown in Fig. 1, and contours are drawn for a gain value of 0.5 .

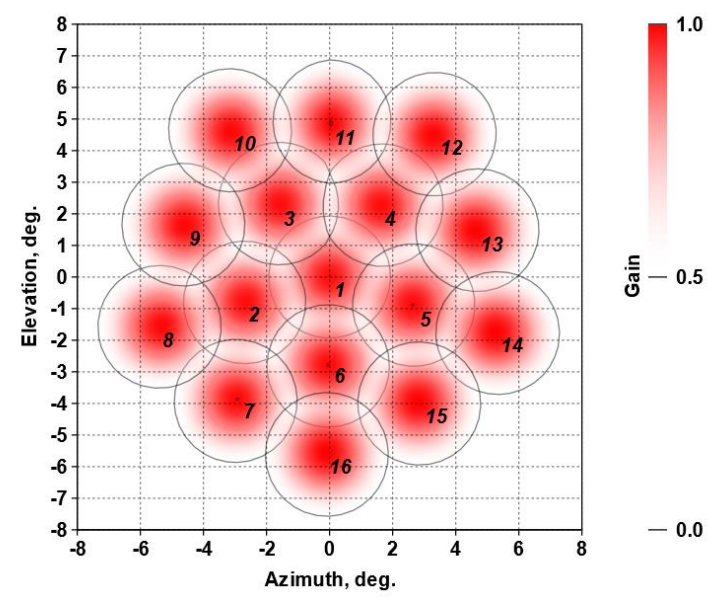

Fig. 1. Radiation pattern of a simulated antenna. 
The set of signal amplitudes recorded in the antenna beams is given by expression:

$$
A_{i}=A_{0} d_{i}\left(\varphi_{0}, \theta_{0}\right)+A_{N i},
$$

where $A_{i}$ is the amplitude of radiation received by $i$-th RP beam, $A_{0}$ is radio source amplitude, $A_{N i}$ is noise amplitude in $i$-th RP beam, $d_{i}$ is RP of $i$-th beam, $\left(\varphi_{i}, \theta_{i}\right)$ is radio source bearing.

It is assumed that the source signal contains additive white Gaussian noise. According to [7], $\Delta A_{i}$ obeys the Rayleigh distribution, the width of which is related to the signal-tonoise ratio (SNR) in the communication channel. The relation is given by expression:

$$
\sigma=\frac{A}{\sqrt{2}} 10^{-\frac{S N R_{o u t}}{20}}
$$

where $S N R_{\text {out }}$ is SNR at the output of the receiving system, $\sigma$ is Rayleigh distribution width parameter.

Using narrow beamwidth antenna allows to suppress noise received from directions that are greatly differ from the direction of the electrical axis which significantly increases the SNR. The increase of SNR $(\triangle S N R)$ can be calculated as follows:

$$
\Delta S N R=10 \lg \frac{d^{2}{ }_{\text {max }} \int d \varphi d \theta}{\int d^{2}(\varphi, \theta) d \varphi d \theta} .
$$

where $d_{\max }$ is the maximum value of radiation pattern.

Evaluation by formula (3) gives an approximate value of $\triangle S N R$ equal to $33 \mathrm{~dB}$. During the simulation, noise samples were added to the signal amplitudes, the magnitude of which obeys the Rayleigh distribution with width $\sigma(2)$. The width $\sigma$ calculated considering that $S N R_{\text {out }}=S N R_{\text {in }}+\Delta S N R$.

In passive bearing estimation problems, the most common method is solving a nonlinear equation system for bearing $\left(\varphi_{0} ; \theta_{0}\right)[1,8]$. In this article, it is solved by minimizing the error function (Err) by the simplex optimization method [9]

$$
E r r=\sum_{i=1}^{n-1} \sum_{j=i+1}^{n}\left(\frac{A_{i}-A_{j}}{A_{i}+A_{j}}-\frac{d_{i}\left(\phi_{0}, \theta_{0}\right)-d_{j}\left(\phi_{0}, \theta_{0}\right)}{d_{i}\left(\phi_{0}, \theta_{0}\right)+d_{j}\left(\phi_{0}, \theta_{0}\right)}\right)^{2},
$$

where the amplitudes of the received signal in different channels $A_{i}$ are usually normalized to the maximum amplitude $A_{\max }$ of the received signal for all channels: $\xi_{i}=A_{i} / A_{\max }$, and $n$ is the number of antenna beams.

\section{Estimation of radiation pattern for antenna with a deformed reflector}

To describe the reflector, a Cartesian coordinate system $(\mathrm{x}, \mathrm{y}, \mathrm{z})$ with the origin at the vertex of the paraboloid and the $\mathrm{z}$ axis directed along the focal axis of the paraboloid is used. The $\mathrm{x}$ axis is parallel to the aperture of the reflector and is located in the reference plane of the azimuth angle, the y axis complements the right-handed coordinate system. With one-side heating of the antenna reflector, thermal deformation of the heated half of the reflector occurs. Based on the results obtained for a larger diameter reflector in [10], the deviation of 
the considered reflector from the ideal is about $50 \mathrm{~mm}$. In this case, the points located closer to the edge of the reflector are shifted stronger than the points located closer to the center of the reflector. The following deformation model was chosen in the article:

$$
x \rightarrow x ; \quad y \rightarrow(1+\alpha) y, y>0 ; \quad y \rightarrow y, y<0 ; \quad z \rightarrow z
$$

where $\alpha$ is the linear deformation coefficient (shows how much the heated side of the reflector was deformed relative to the initial state). In the article, the parameter $\alpha$ is considered known.

The displacement of $\sim 10^{-2} \mathrm{~m}$ can be considered small compared to the reflector radius of $\sim 1 \mathrm{~m}$, therefore the radiation pattern profile remained unchanged, but the antenna electric axis is shifted. When the antenna reflector is stretched the focus position changes and the antenna irradiators are shifted relative to the focus. According to the antenna theory [4], it leads to the electric axis displacement, and since deformation occurs along the $x$ axis; displacement occurs only along the azimuth angle. Mathematically this is expressed as follows:

$$
d_{d e f}(\varphi, \theta)=d_{i d}\left(\varphi-\varphi_{\Delta}, \theta\right)
$$

where $d_{d e f}$ is deformed antenna radiation pattern, $d_{i d}$ is undeformed antenna radiation pattern, $\varphi_{\Delta}$ is the deformed antenna electrical axis displacement relative to the position of the undeformed antenna electrical axis.

Due to the smallness of the reflector deformation, it is proposed to assume that the electrical axis displacement linearly depends on the coefficient $\alpha$

$$
\varphi_{\Delta}=b \alpha
$$

The free term of linear dependence equals zero, due to the absence of the considered displacement at zero reflector deformation. To check wherever the dependence of $\varphi_{\Delta}$ on $\alpha$ is linear, it is necessary to use the linear correlation coefficient [7]. If the linear correlation coefficient is close to 1 , then the dependence is considered linear and linear regression can be applied. In this case, the coefficient $b$ is estimated as follows [7]:

$$
b=\frac{\sum_{i=1}^{K}\left(\varphi_{\Delta i}-\left\langle\varphi_{\Delta}\right\rangle\right)\left(\alpha_{i}-\langle\alpha\rangle\right)}{\left\langle\alpha^{2}\right\rangle-\langle\alpha\rangle^{2}} .
$$

where $t_{\alpha, K-2}$ is Student coefficient for $K-2$ degrees of freedom, angle brackets indicate averaging.

The parameter $b$ is different for different antennas, and for each of them it is necessary to carry out a procedure for determining its value. When using long-focus antennas, the electric axis displacements of various beams relative to the paraboloid focal axis are small. Therefore, the coefficient $b$ can be considered the same for all antenna beams.

It is possible to estimate the radiation pattern of antenna using formulas (6) and (7). The proposed method does not require analytical recalculation of the radiation pattern, and the correction calculation consists from several linear operations, which leads to computational efficiency. Before applying the method, it is necessary to carry out the procedure for determining the parameter $b$ once. 


\section{Simulation}

\subsection{Linear displacement model verification}

As noted earlier, before using the model of linear dependence of the electric axis displacement on the reflector deformation, it is necessary to verify its feasibility. It is assumed in the work, if the absolute value of the linear correlation coefficient exceeds 0.999 , then the linearity assumption is valid.

For simulation, an antenna with a reflector aperture diameter of $D=10 \mathrm{~m}$ and focal length of $F=7 \mathrm{~m}$ was considered. Also, typical 16-irradiator configuration was used for simulation. Irradiator frequency $f$ was taken as $8 \mathrm{GHz}$. To calculate the radiation pattern, the reflective point method was used; the antenna was discretized in such a way that the points were located no further than half the wavelength from each other. The Fig. 2 shows the results of calculating the electric axis displacement for different coefficients $\alpha$ from 0 up to 0.01 (which corresponds to a deformation from 0 to $50 \mathrm{~mm}$ ).

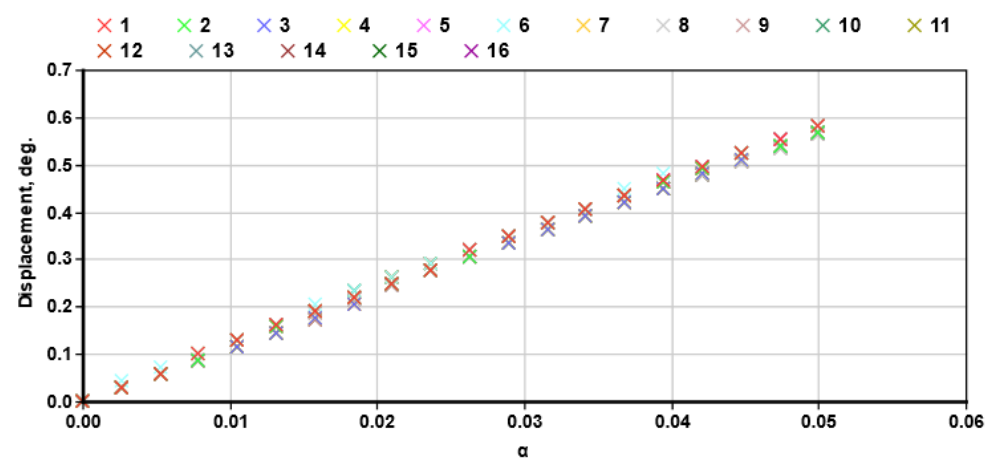

Fig. 2. Relation between the electric axis displacements and the reflector deformation.

For the obtained relation, the values of the linear correlation coefficients $R$, coefficients $b(8)$ and its absolute error $\Delta b$ (the error was estimated according to the rules for estimating the error of the least square coefficients [7]). For calculation, a confidence probability of 0.99 was taken. The results are presented in table 2 .

Table 2. The values of the coefficients of linear correlation and linear regression.

\begin{tabular}{|c|c|c|c|c|c|c|c|c|c|c|c|}
\hline № & $R$ & $\begin{array}{c}b 10^{-1} \\
\text { deg. }\end{array}$ & № & $R$ & $\begin{array}{c}b 10^{-1}, \\
\text { deg. }\end{array}$ & № & $R$ & $\begin{array}{c}b 10^{-1}, \\
\text { deg. }\end{array}$ & № & $R$ & $\begin{array}{c}b 10^{-1} \\
\text { deg. }\end{array}$ \\
\hline $\mathbf{1}$ & 0.9999 & $120 \pm 9$ & $\mathbf{5}$ & 0.9999 & $121 \pm 11$ & $\mathbf{9}$ & 0.9999 & $118 \pm 6$ & $\mathbf{1 3}$ & 0.9999 & $118 \pm 8$ \\
\hline $\mathbf{2}$ & 0.9999 & $121 \pm 8$ & $\mathbf{6}$ & 0.9999 & $122 \pm 7$ & $\mathbf{1 0}$ & 0.9999 & $114 \pm 7$ & $\mathbf{1 4}$ & 0.9999 & $121 \pm 8$ \\
\hline $\mathbf{3}$ & 0.9999 & $117 \pm 8$ & $\mathbf{7}$ & 0.9999 & $124 \pm 8$ & $\mathbf{1 1}$ & 0.9999 & $114 \pm 7$ & $\mathbf{1 5}$ & 0.9999 & $124 \pm 6$ \\
\hline $\mathbf{4}$ & 0.9999 & $117 \pm 5$ & $\mathbf{8}$ & 0.9999 & $121 \pm 4$ & $\mathbf{1 2}$ & 0.9999 & $114 \pm 8$ & $\mathbf{1 6}$ & 0.9999 & $125 \pm 11$ \\
\hline
\end{tabular}

The calculated correlation coefficients allow to conclude that the linear regression model is valid for the considered relation. As expected the value of $b$ differs slightly for different beams, so for the rest of the paper the coefficient $b$ is assumed to be the same for all beams. The average value is $b=12.0 \pm 0.2$ degrees (the error was estimated according to the rules for estimating the error of indirect measurements [7]). 


\subsection{The probability of determining a bearing with error less than fixed threshold}

To estimate the bearing, the simplex optimization method of the error function, presented in Sec. 2, was used. The simplex method is iterative and initial approximation is taken as a node with minimal functional value from uniform grid $(10 \times 10$ points $)$. Of practical interest is the estimating of a bearing with an error that less than fixed threshold. In the article, an error of 0.2 degrees is assumed as the threshold. The probability of bearing estimation under this condition can be used as a quality criterion of the solving algorithm. For each state of the antenna system, 10000 experiments were performed to determine the probability. When registering the amplitudes, the SNR at the input of the antenna system is fixed and Rayleigh distributed noise samples are added. For each experiment location of the radio source was sampled randomly from uniform distribution over simulation area of $\varphi \in[-8 ; 8]$ degrees, $\theta \in[-8 ; 8]$ degrees.

The relation between probability of determining the bearing with an error less than threshold of 0.2 degrees and SNR at the input of the antenna system is shown in Fig. 3. The green chart is obtained using the initial RP. The blue chart is obtained using the RP calculated by the reflective point method. The latter chart is obtained by computationally inefficient method, but considers the reflector deformation as accurately as possible. The gray chart is obtained using correction by the model of the linear beam displacement.

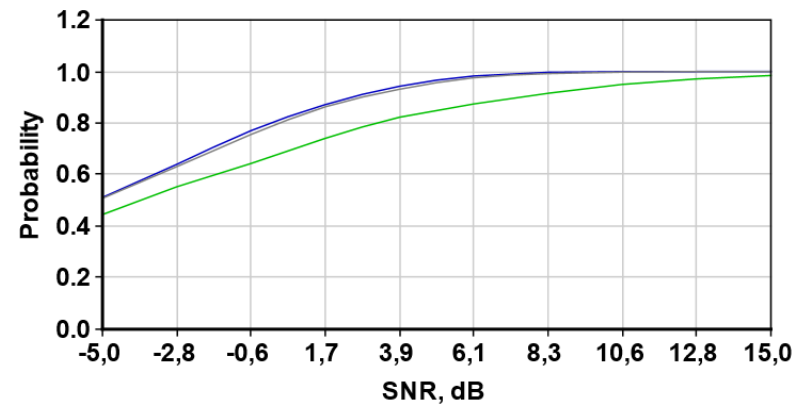

Fig. 3. The relation between probability of determining the bearing with an error less than threshold of 0.2 degrees and SNR.

In practice, it is important to estimate the bearing with probability of error being within the acceptable range close to 1 . It follows from the chart that the proposed model can be used at lower SNR without reducing the probability. For example, with a probability of 0.95 , it is possible to work with signals at SNR approximately $4 \mathrm{~dB}$ lower. Also, with a fixed SNR, the probability increases. For example, with an SNR of $6 \mathrm{~dB}$, this probability increases by 0.1 .

\section{Conclusion}

An algorithm for updating the radiation pattern is proposed in the article. The algorithm allows to consider antenna reflector deformation, for example, due to its uneven heating. It is shown that an electric axis displacement due to the reflector deformation linearly depends on the deformation value. This makes it possible to use proposed method, which makes it possible to work with signals at lower SNR with fixed probability of determining the bearing with error less than fixed threshold. The result of applying the method can also be interpreted as an increase in the probability for a fixed SNR. 


\section{References}

1. A.I. Leonov, K.I. Fomichev, Monopulse radiolocation (Moscow, Radio i svyaz, 1984, in Russian)

2. A.A. Loginov, O.A. Morozov, M.Y. Semenova, Algorithms for improving the accuracy of bearing estimation in the problem of amplitude monopulse passive location, Vestnik nizhegorodskogo universiteta im. N.I. Lobachevskogo 5, 358 (2010, in Russian)

3. M. G. Mathylenko, M. O. Dorofeev, E. V. Bikeev, A. A. Alexeenko, Control system of large-sized transformed antenna geometry, Reshetnevsie chteniya, 194 (2013, in Russian)

4. A.L. Drabkin, V.L. Zuzenko, A.G. Kislov, Antenna feeder devices (Moscow, Sovietskoe radio, 1974)

5. E.V. Bikeev, E.N. Yakimov, M.G. Matilenko, G.P. Titov, The method of compensation of construction deformation for large spacecraft antenna, Sibirsii Gosudarstvennyi Aerokosmicheskii Universitet imeni Akafemika M.F. Reshetneva. Vestnik 17(3), 673 (2016)

6. D.V. Dyatlov, N.R. Halimov, V.P. Sidorchuk, Detection of phased array not working on radiation, Zhurnal radioelectroniki 1, (2014, in Russian)

7. A.N. Zaidel, Measurement error of physical quantities (Leningrad, Nauka, 1985)

8. A.A. Loginov, O.A. Morozov, M.Y. Semenova, V.R. Fidelman, Method for estimating the number of radiation sources in the problem of the amplitude monopulse direction finding, Radiophysics and Quantum Electronics 56(7), 456 (2013)

9. A.P. Dambrauskas, Simplex search (Energiya, 1979)

10. V.S. Ponomarev, S.V. Ponomarev, V.I. Khalimanovich, Thermal-structural analysis of large mesh reflector for space application, Sibirsii Gosudarstvennyi Aerokosmicheskii Universitet imeni Akafemika M.F. Reshetneva. Vestnik 17(2), 343 (2016) 